Arts et Savoirs

et Savoirs

3 | 2013

L'adaptation comique

\title{
Un parodiste moderne
}

L'œuvre de David Godbold

Valérie Morisson

\section{(2) OpenEdition}

Journals

Édition électronique

URL : http://journals.openedition.org/aes/415

DOI : $10.4000 /$ aes. 415

ISSN : 2258-093X

Éditeur

Laboratoire LISAA

Référence électronique

Valérie Morisson, "Un parodiste moderne », Arts et Savoirs [En ligne], 3 | 2013, mis en ligne le 15 février 2015, consulté le 30 avril 2019. URL : http://journals.openedition.org/aes/415; DOI : 10.4000/aes.415

Ce document a été généré automatiquement le 30 avril 2019

Centre de recherche LISAA (Littératures SAvoirs et Arts) 


\title{
Un parodiste moderne
}

\author{
L'œuvre de David Godbold
}

\section{Valérie Morisson}

1 Si le genre comique, la parodie et l'esthétique burlesque sont avant tout des catégories littéraires, les beaux-arts ne rechignent guère à puiser dans ces registres. Ainsi, le travail de David Godbold emprunte au burlesque ses décalages en cascade et à la parodie ses clins d'œil appuyés. Dessinateur talentueux, il joue sur l'articulation comique entre le texte et l'image par le biais de superpositions qui, loin de mener à une analogie entre le visuel et le textuel, illustrent l'irréductibilité de l'un à l'autre. David Godbold est né en 1961, à Norwich en Angleterre. Après avoir étudié à la Norwich School of Art and Design, voyagé dans divers pays, il s'est s'installé à Dublin, où il enseigne au National College of Art and Design. Le nom de l'artiste, ainsi que l'exposition itinérante réalisée en 2007, The Unreliable Narrator (ill. 1), mettent d'emblée le spectateur sur la piste de la parodie. Les montages composant cette exposition sont à la croisée de genres anciens, ceux de la parodie et du burlesque, et du postmodernisme, mouvement contemporain que l'artiste connait bien d'un point de vue tant théorique que plastique. Des personnages populaires extraits de la culture de masse, des objets de la vie courante, ou bien des textes apocryphes font irruption dans des compositions à l'encre qui parodient le Grand Art. L'incongruité des rapprochements est frappante, l'impertinence, manifeste. En effet, si David Godbold imite la grande peinture religieuse en réalisant des esquisses magistrales, les légendes, bulles ou ready-made visuels et textuels qu'il ajoute au moyen d'un calque font de lui un parodiste postmoderne, héritier de Dada et du Pop art. En superposant des résidus de la culture de masse et des dessins maniéristes aux accents baroques, Godbold crée un effet comique dont on se propose de cerner les ressorts. 


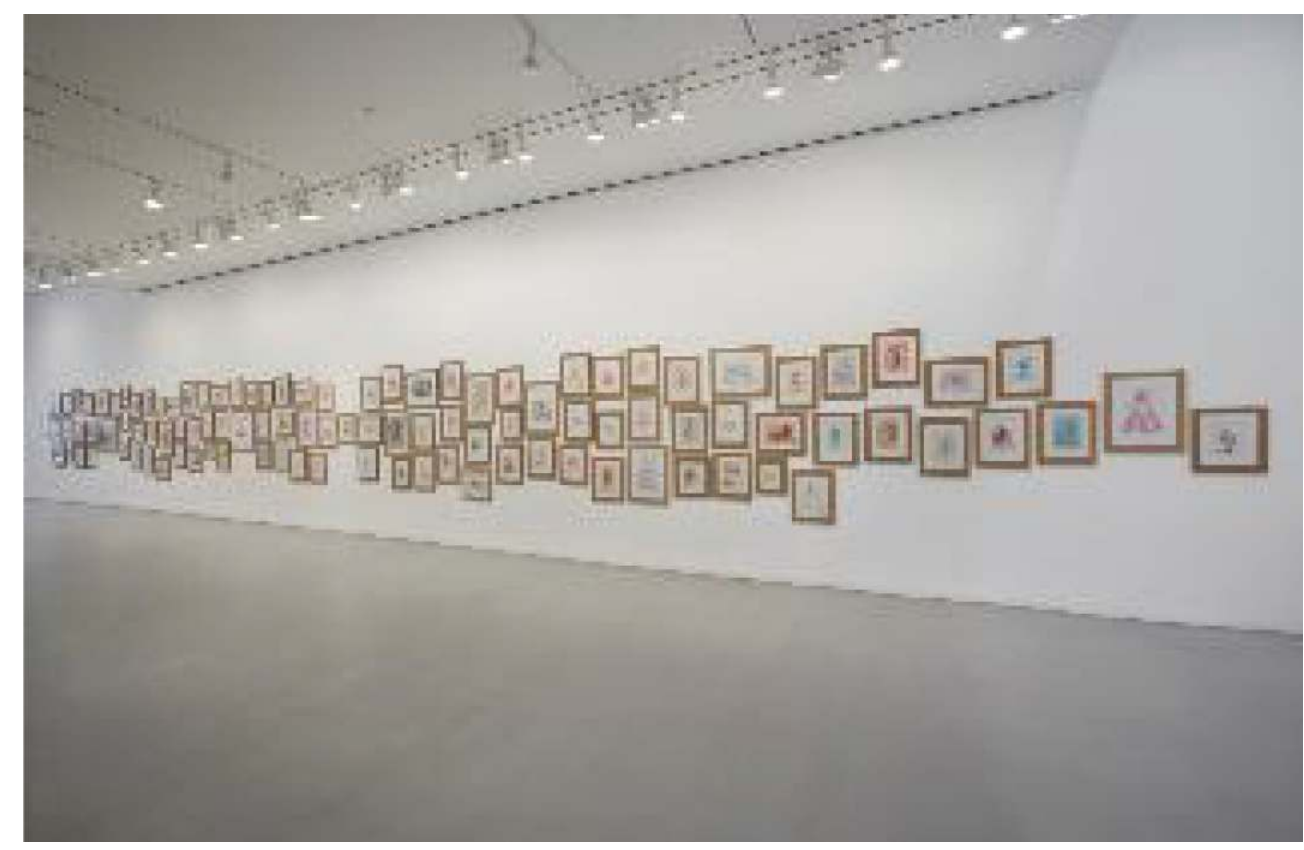

Avec l'aimable permission de l'artiste et de la galerie Mitchell-Innes \& Nash.

\section{Un comique à distance}

Avant de s'aventurer plus avant sur le terrain de la parodie, notons de manière préliminaire que toute parodie suppose que l'hypotexte, pour reprendre le terme de Genette -ou l'œuvre source à laquelle l'auteur emprunte - soit identifié comme appartenant à un genre. En effet, « la parodie est un instrument d'écriture reposant sur la manipulation visible de traits textuels, et le plus sûr moyen de faire percevoir un genre : l'écrivain s'étant livré à une opération de généralisation [...] puise à un répertoire et le lecteur y reconnaît à l'état de caricature un modèle générique. $»^{1}$ Pour qu'il y ait parodie, et effet comique, il faut que le spectateur identifie le lien entre l'image source et l'image parodique «empruntante» (ou hyper-icône qui serait un équivalent visuel de l'hypertexte). Toute parodie suppose conséquemment une connivence intellectuelle entre le lecteur et l'auteur. Le visiteur de The Unreliable Narrator reconnaîtra aisément les hypo-icônes, pour décliner, sans le parodier, le lexique genettien: Godbold prend un malin plaisir à contrefaire les paysages de Constable, les dessins à l'encre des maîtres italiens ou les personnages cubistes de Picasso, autant d'œuvres qui, si elles ne sont pas individuellement identifiées, sont néanmoins reconnues comme chefs-d'œuvre. Dans les nombreux cas où l'hypotexte, ou hypo-icône, est religieux, le langage visuel de la Contreréforme est caractérisé pour être parodié : gestes amples, visages empreints de pathos, circonvolutions des draperies, etc. L'utilisation de l'encre, technique classique, tout comme l'abondance de signes religieux, suffisent à évoquer un genre artistique noble. Rappelons d'ailleurs que pour Michele Hannoosh, la parodie « crée l'original dans l'esprit du lecteur, le construisant dans l'acte même de le transformer'². C'est le cas de plusieurs œuvres de David Godbold. 
Ces œuvres ont une efficacité comique dans la mesure où elles permettent une distanciation qui, comme le montre Jean Emelina, est essentielle au comique : il ne peut y avoir, dans ce cas, aucune identification du spectateur aux personnages qui sont l'objet d'irrévérence ${ }^{3}$. "Qu'il s'agisse du vécu ou de la fiction, le rire exige donc une position de spectateur et un non-engagement ${ }^{4}$. " Les situations évoquées par Godbold réalisent les trois conditions de l'émergence du comique isolées par Emelina : la distance, l'anomalie et l'abolition des effets indésirables de l'action. La distance, essentielle au comique, est parfois temporelle (c'est le cas des parodies d'œuvres de la Contre-Réforme) parfois sociale (dans le cas des œuvres se moquant de l'art et de la culture des élites). L'anomalie qui provoque le comique suppose une norme explicite qui doit être connue du spectateur. Dans le cas du travail de Godbold, cette norme est l'unité et l'unicité de l'œuvre. Il transgresse ces normes en empruntant et transformant ces objets culturels. Enfin, les mutations du modèle n'ont aucun effet négatif sur le spectateur et ne sont donc nullement tragiques.

4 À titre introductif, notons également qu'à la différence du pastiche, la parodie implique fondamentalement une relation critique à l'objet parodié. Bakhtine parle même de destruction parodique lorsque la parodie réduit à l'absurde certains traits caractéristiques. ${ }^{5}$. Il a démontré que la parodie «engageait des postures culturelles, sociales, politiques ${ }^{6} »$, des traits qu'il conviendra de dégager dans l'œuvre de Godbold. L'imitation parodique s'accompagne de charge, de caricature, ou bien ridiculise une forme ancienne et vénérée par des familiarités, des trivialités, des anachronismes. Ces décalages sont légion dans l'œuvre de Godbold.

\section{L'hybride au cœur de la parodie}

5 La parodie est, par nature, hybride. Or, en juxtaposant de façon incongrue des images et des textes, Godbold multiplie les procédés d'hybridation. L'unité de l'image est perturbée par l'irruption de textes ; les éléments textuels eux-mêmes sont disparates, appartenant à des registres incompatibles; enfin, les dessins maniéristes sont associés à des signes visuels qui les désacralisent.

6 Plusieurs types de textes s'immiscent dans les compositions de David Godbold. Des motssignes surgissent derrière l'image et sont perçus presque simultanément au dessin. Des mots ou chiffres hantent l'image plus qu'ils ne l'envahissent. Des légendes dactylographiées, parfois reprises dans le titre de l'œuvre, forcent le spectateur à aller et venir entre le texte et l'image. Les montages de Godbold, comme des bandes dessinées, ne présentent pas deux sources disjointes et stables mais constituent un dispositif dynamique de double écriture :

Dans la mesure où l'on peut « voir » l'image et les mots simultanément, la présence de l'un nécessite l'absence de l'autre, créant un jeu de différences irrésolubles et continues entre les deux formes textuelles... La signification et la stabilité du sens sont constamment différées alors que l'œil ... saute des mots aux images, effectuant des circonvolutions et des zigzags et interrompant fréquemment le processus entier pour reparcourir l'information d'une manière nouvelle ${ }^{7}$.

7 Bien que décrivant habilement l'opération de déchiffrage à laquelle le spectateur doit se livrer devant les montages de Godbold, les propos de Schmitt s'inscrivent dans une tendance critique qui assimile volontiers l'image à un texte, comme le fait notamment Roland Barthes ${ }^{8}$. Or, si certaines images se déchiffrent à la manière d'un texte, 
consolidant ainsi la doctrine de l'ut pictura poesis, les vignettes ou extraits visuels de Godbold restent autant fragmentaires qu'emblématiques et appellent davantage une lecture immédiate. Ce qui intéresse Godbold, ce sont les zones de frottements, les points d'achoppement entre l'image et le texte.

8 L'hétérogénéité de l'œuvre induite par la co-présence du visuel et du verbal se double d'une hétérogénéité générique radicale propre à la parodie. En effet, à l'intérieur d'une même composition, sont intégrés des registres incompatibles. Dans Free leg wax..., une gravure à l'encre brune représentant une femme dont le corps à demi nu émerge d'un arbre feuillu, deux registres textuels différents sont superposés à l'image: le registre sérieux de la légende dactylographiée mais inattendue (Are you finding what I'm thinkin (sic.)) et le registre décalé du slogan publicitaire recopié au crayon en arrière-plan (Free leg wax for every haircut). Les deux légendes, tout aussi incongrues l'une que l'autre, conduisent à une relecture de l'image source. De la même manière, dans I like to eat..., Godbold mêle un texte prosaïque évoquant de manière banale les goûts alimentaires du personnage (I like to eat a lot of chocolate) et un texte pseudo théorique véhiculant une appréciation esthétique (Allergic to any relapse into magic, art is part of the disenchantment of the world). Cet entrechoquement du trivial et du profond, du concret et de l'abstrait, du physiologique et du philosophique est depuis longtemps, dans la littérature, source de comique. La parodie, telle que la comprend Bakhtine, c'est-à-dire comme une pratique d'imitation ou de transformation caricaturale, implique un plurilinguisme, l'«évocation parodique de presque toutes les couches du langage littéraire parlé et écrit de son temps9." Bakhtine appelle hybridation «le mélange de deux langages sociaux à l'intérieur d'un seul énoncé ${ }^{10}$. » Godbold affuble fréquemment les reproductions d'images religieuses de légendes grossières. Dans The Conversion (ill. 2), le lexique scatologique de la légende (I'm so happy I could shit) dénature l'image qui, elle, met en scène un ange et une âme perdue. Michele Hannoosh définit la parodie comme «le re-travail et la transformation comiques d'un autre texte par distorsion de ses traits caractéristiques. » Cette transformation résulte de la «distorsion de traits stylistiques, l'inversion de valeurs, la transposition dans un contexte nouveau, incongru et souvent trivial, etc. ${ }^{11} »$ Les parodies irrévérencieuses de Godbold ont la particularité de mêler texte et image, ce qui accentue le comique dû à l'incongru. Selon Jean-Charles Chabanne «La réaction comique (sur toute l'échelle de ses variantes, de l'amusement au rire), serait provoquée par un contraste brutal et rapide entre les attentes perceptives et la perception elle-même. On attendrait quelque chose qui soit conforme à des habitudes anticipatives, et viendrait quelque chose d'absolument inattendu, qui ne fait pas sens selon nos prévisions. $»^{12}$ Lorsqu'il est dû à l'incongruité, l'effet comique peut provenir d'une focalisation inattendue. Dans Infamy, infamy, un martyr ressemblant au Christ luimême prononce ces mots : "infamy, infamy everyone's got it in for me ». L'absence de guillemets dans l'œuvre laisse planer un doute sur la focalisation, d'autant que le registre familier détone par rapport au langage attendu de la part d'un saint chrétien. Dans Friday is healthy lunch day, le spectateur est libre d'attribuer ces mots, encadrés de rouge, à Saint Marc s'adressant au Christ même si aucun dispositif sémiotique particulier ne l'y autorise. Les éléments textuels demeurent fréquemment intradiégétiques. Néanmoins, l'absence de guillemets ou bien la présence de pronoms sans référence dans les légendes ou les textes en arrière-plan ébranlent le dispositif de lecture. Quant aux mots figurant dans les bulles superposées aux dessins, ils trahissent les personnages qui ne se savent pas écoutés. Godbold adapte sciemment l'ironie dramatique à ses dispositifs hybrides. Le 
décalage de registre laisse constamment planer un doute sur la focalisation et sur l'identité du personnage. Le titre de l'exposition, The Unreliable Narrator, a d'ailleurs de forts relents postmodernes et se trouve réfracté dans l'instabilité de la focalisation que nous venons d'évoquer.

III. 2 : David Godbold, The conversion (servisi), Kerlin, 2002

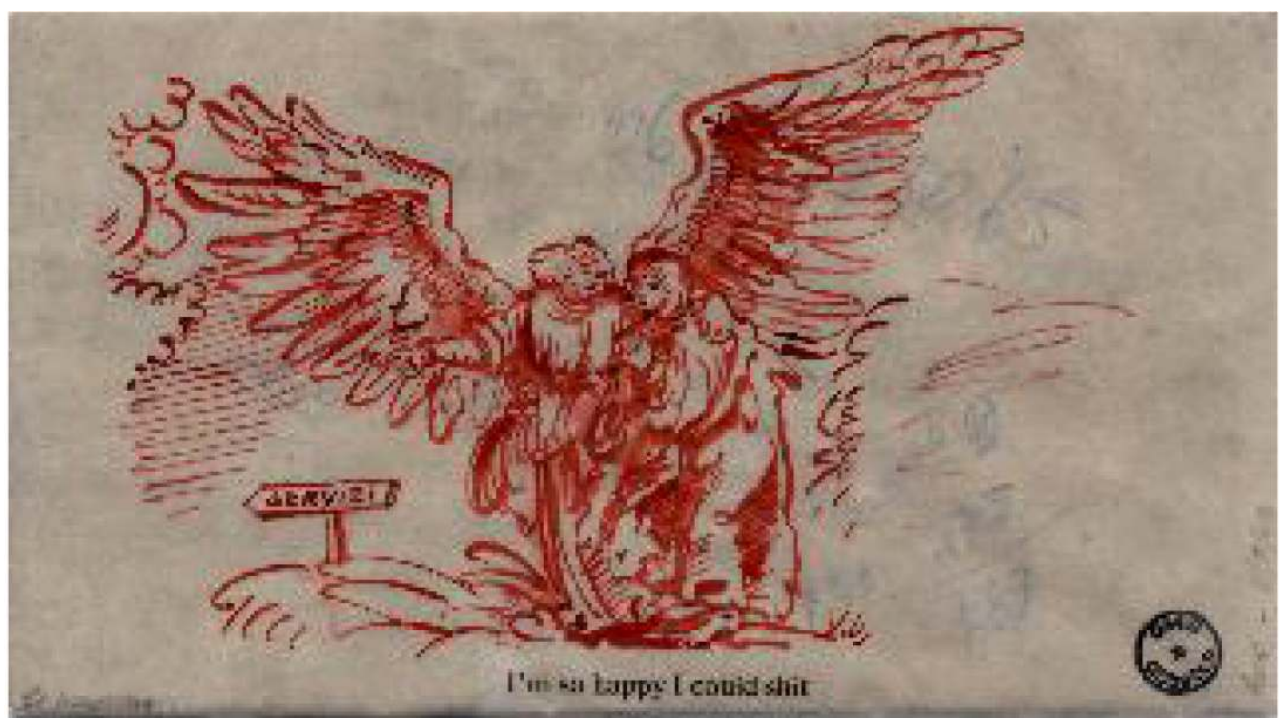

Encre et impression sur papier-calque et ready-made, $10 \times 17.5$ cm, DG20202.

Avec l'aimable permission de l'artiste et de la galerie Kerlin.

Dans plusieurs œuvres, l'hybridation parodique résulte de la superposition de signes visuels n'appartenant pas au même registre iconique : un chef d'œuvre et un graffiti par exemple. Dans I believe in days ahead, dont le titre est ironique, Godbold superpose une reproduction à l'encre verte figurant une vierge à l'enfant et un tableau enregistrant des dépenses mensuelles. Le sacré s'oppose au prosaïque, le spirituel au matériel, l'affection à l'exercice comptable, le grand art à de triviales notations. "I am interested in the conflation of grand themes and daily minutiae ", avoue David Godbold ${ }^{13}$. Dans Head in a Bag, l'artiste revisite le thème de Judith présentant la tête d'Holopherne mais désacralise le sujet en travestissant le titre, Head in a Bag étant exempt de toute référence culturelle. Il utilise des encres de couleurs variées et vives, notamment du rose et de l'orange, pour déclasser son dessin. Enfin, de manière à parfaire la parodie, il glisse des signes visuels hétéroclites (suites arithmétiques et dessins d'adolescents) derrière le calque où sont représentées les figures. La superposition des deux représentations, y compris sur les corps des personnages, ainsi que la discordance entre la nature tragique de la scène et la fantaisie des coloris sont cocasses puisqu'elles induisent un changement de registre et de genre. Pour autant, il n'y a nulle caricature mais bien une parodie qui met en tension l'hypotexte et l'hypertexte sans dénigrer absolument l'image source. Margaret Rose insiste sur l'ambivalence de la relation entre l'œuvre source et l'œuvre parodique critique : il existerait simultanément un désir d'imitation et un souci critique ${ }^{14}$. Chez Godbold, cette tension se fait sentir par la minutie du dessin à l'encre, le soin apporté à la transcription des scènes et le plaisir technique évident qu'a l'artiste à contrefaire les chefs-d'œuvre. De manière concomitante, le caractère transcendantal du débat de la Contre-Réforme, la spiritualité et la morale, sont comme niés par l'irruption de fragments 
de la réalité quotidienne qu'il s'agisse de chiffres comptables, de mots grossiers, de graffitis ou de morceaux de toile cirée...

\section{Des travestissements burlesques} d'une personne ou d'une œuvre d'art, célèbre ou sérieuse, dans le but d'obtenir un effet plaisant ou burlesque ${ }^{15}$. » Les travestissements opérés par David Godbold correspondent au burlesque classique défini par Jean-Marc Defays ${ }^{16}$ et caractérisé par l'inversion des signes et des valeurs, voire par leur négation. «Le burlesque se place en porte-à-faux avec la doxa de l'harmonie et de la convenance esthétique ${ }^{17}$.» Le burlesque est une démarche anti-artistique qui exhibe sa dissonance, sa cacophonie. À la différence du grotesque, il n'implique pas de déformation monstrueuse mais est lié à une conception hiérarchique et cloisonnée des styles.

11 Godbold réintroduit avec espièglerie la culture populaire de la bande dessinée dans le grand art et jette un regard amusé sur les hiérarchies artistiques instituées. Il désacralise les images religieuses, pendant longtemps placées par les académiciens au-dessus de toute autre représentation, et amène le lecteur à regarder les chefs-d'œuvre de la Contreréforme comme des mélodrames populaires empreints de grandiloquence. Il nivelle les représentations en les surimposant. Dans Female Companion Gets Up and Walks Away, Godbold représente à l'encre orange Dieu de profil, les bras levés vers l'angle gauche de l'image et entouré de volutes figurés dans le style baroque. Le dessin est réalisé sur papier calque laissant voir en transparence un écriteau portant la mention CAUTION en rouge. Deux fragments de texte sont tapés à l'encre noire dans les espaces vides autour de Dieu : "There's no need to be nasty " et «I didn't mean it that way ». Le lecteur passe du langage pictural du baroque italien au registre familier de la bande dessinée : voici un saint qui s'est disputé avec sa femme et essaie, tant bien que mal, de la convaincre de rentrer à la maison. Dans Can We Kill It ? (ill. 3), ce sont quatre gladiateurs romains dessinés à l'encre verte qui sont affublés de bulles de bandes dessinées, ce qui suffit à provoquer un effet comique dû à l'anachronisme et à la conflagration d'un genre dit noble, celui de la gravure, et d'un genre populaire. 


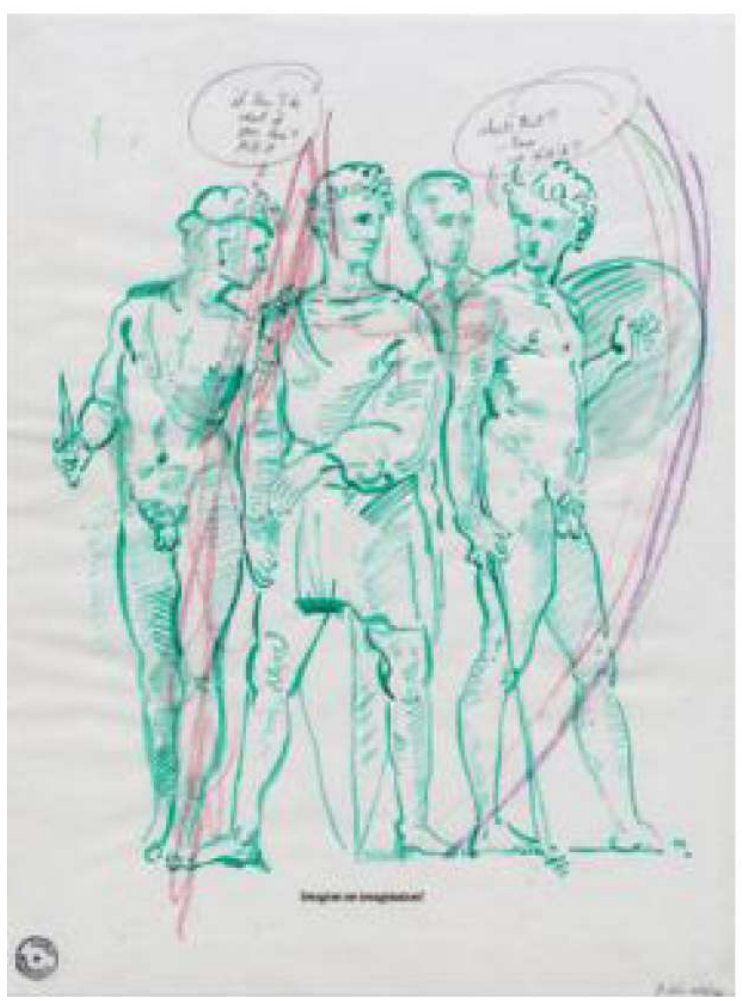

Encre sur papier-calque, $27.7 \times 20.5 \mathrm{~cm}$.

Avec l'aimable permission de l'artiste.

Si l'introduction d'éléments de culture populaire au sein du grand art était une revendication des artistes associés au Pop Art, dans le travail de Godbold grand art et culture populaire interagissent de manière dialogique. Ainsi, l'esthétique kitsch qui affleure dans certaines œuvres invite le spectateur à s'interroger autant sur le fétichisme de nos sociétés de consommation que sur l'illusion sentimentale des peintures religieuses. Le kitsch, écrit Guy Scarpetta, "c'est l'art du mauvais goût, stéréotypé, la beauté artificielle, réduite à son emphase ou à ses apparences ${ }^{18}$. $"$ De manière générale, les dessins monochromes à l'encre de Godbold rappellent les anciens almanachs du XVIe siècle, les illustrations à l'encre de couleur des partitions de chants religieux, les gravures populaires, les bibles en images ou bibles illustrées. La présentation des œuvres de petite taille dans la galerie vise à évoquer une collection de vignettes plus qu'une série d'œuvres majeures. L'accrochage appuie ainsi le propos de l'artiste: les œuvres deviennent des fragments culturels multiples et variés s'accommodant d'un certain kitsch. La superposition parodique d'œuvres anciennes développant une rhétorique de l'emphase et du sentimentalisme et de décors kitsch des années 1970 interroge donc le sentimentalisme complaisant, l'orientation mercantile de l'industrie culturelle, le goût pour l'art de pacotille, la profusion des éléments décoratifs et l'accumulation, la fonction sociale et affective des simulacres de culture quelle que soit la période considérée.

Dans "Poétique du burlesque», Dominique Bertrand isole certains traits du genre: l'outrance, la discordance, la mystification, la surenchère, la démesure, l'exagération bouffonne, la truculence et la fantaisie verbale ${ }^{19}$. Le burlesque développe des liens intimes avec la parodie puisqu'il est une réécriture en style vulgaire: il consiste à parler en 
termes grossiers de choses sérieuses et repose sur le travestissement. Ce faisant, il se rapproche du grotesque et de l'absurde. Souvent qualifié de bas, car il verse volontiers dans un registre grossier, vulgaire et scatologique, il est accusé d'être de mauvais goût. Dans de nombreuses compositions, Godbold puise dans le registre sexuel: Teenage Sex Priest, Testacles sont des titres évocateurs. Dans Meow ! the Miracle of the Espresso Ring (ill. 4), sous les figures saintes, est inscrit: "Heaven preserve us from sea storm and annoying fucking arseholes ». Le titre provient de la trace ronde laissée par la tasse d'espresso qui se métamorphose en auréole. Autre source de comique à l'œuvre ici, la présence anachronique du logo et de l'adresse électronique d'Holiday Inn au bas de la page. Dans Stating the Obvious, la désacralisation de la vierge à l'enfant est radicale puisque le mot «fucking» lui est clairement apposé. «Le burlesque transgresse tous les tabous, revendiquant le droit à rire de tout, y compris de la mort et du sacré20. » Godbold est un artiste sacrilège inscrivant sans ambages son œuvre dans le champ du burlesque : «Le burlesque dit ce qui ne se dit pas, fait ce qui ne se fait pas, transgresse ouvertement l'interdit. Il est à l'opposé de la pudeur, de l'allusion, de l'euphémisme et de la litote ", analyse Emelina ${ }^{21}$. Les parodies burlesques de Godbold sont fantaisistes et ludiques : «Le burlesque [...] défigure, disloque, déréalise, pousse à une invention toujours recommencée et toujours extrême. Il est le royaume de la «fantaisie » au sens anglo-saxon du terme («fantasy ») $)^{22}$.» Le burlesque opère des renversements, bouleverse la hiérarchie du monde de manière inconvenante et engendre une "désorganisation préméditée et savourée du réel dans tous les domaines ${ }^{23}$. " Mais, comme nous le verrons à travers les œuvres de Godbold, le burlesque fait parfois grincer les dents et se rapproche de l'humour noir. Il entretient, de fait, des liens avec le persiflage philosophique à des périodes de crises culturelles, sociales ou politiques ${ }^{24}$.

\section{Une parodie burlesque et... postmoderne}

14 Comme la parodie, le burlesque opère sur une modalité intertextuelle mais serait " une parodie qui s'exhibe, qui se projette, bref, une auto-parodie $»^{25}$ capable de dérision vis-àvis d'elle-même. En effet, l'auteur burlesque intervient, commente, ridiculise ses intentions. Le burlesque implique « une vision de l'art (de la littérature, de la peinture, du cinéma) libre, lucide, ludique qu'il partage précisément avec le baroque, mais aussi avec certains aspects du symbolisme, du surréalisme, du postmodernisme » avance Jean-Marc Defays $^{26}$. Les focalisations erratiques, les emprunts et le décloisonnement des hiérarchies que l'on a noté dans les compositions de Godbold font de lui un artiste parodique postmoderne. En outre, son travail est réflexif dans la mesure où plusieurs compositions parodient l'art lui-même ou sont empreintes d'autodérision.

15 Le pacte postmoderne implicite dans le titre de l'exposition, The Unreliable Narrator, n'est pas démenti: Godbold s'avère en plusieurs points un parodiste postmoderne. Premièrement, dans l'œuvre postmoderne, il n'y a plus d'unité imposée dans le medium :

La pratique [du postmodernisme] est définie non pas par rapport à un medium donné mais davantage selon les opérations logiques effectuées sur une série de termes culturels pour lesquels tout medium - la photographie, les livres, les lignes sur les murs ou la sculpture elle-même - peut être utilisé27.

Dans les recompositions de Godbold, les frontières artistiques sont sciemment transgressées. L'hybridité vient remplacer la pureté qui présidait à l'unité stylistique de l'œuvre et au choix d'une manière picturale. Deuxièmement, l'artiste imite à plusieurs reprises les cachets d'authentification de l'institution muséale ou l'inclusion de faux 
numéros d'archives. Il amorce ainsi une réflexion sur les caractéristiques sémiotiques qui distinguent l'œuvre d'autres signes visuels, une démarche critique qui n'est pas sans rappeler les positions de Roland Barthes concernant la prééminence des conventions sur l'inspiration personnelle dans l'élaboration de l'œuvre ou celles de Michel Foucault reliant, quant à lui, la fonction de l'auteur aux systèmes institutionnels et légaux qui délimitent, déterminent et articulent les domaines du discours ${ }^{28}$. L'approche structuraliste de la création s'enrichit plus tard de la réflexion postmoderne sur l'unicité et l'originalité comme illusions perdues. Ayant peut-être en tête le Model for Amateurs de Warhol, où l'on pouvait colorier un paysage en respectant un code chromatique, Godbold produisit en 1998 un livret d'instruction détaillé pour qui souhaiterait réaliser une fresque murale semblable aux siennes ${ }^{29}$. Troisièmement, chez Charles Jencks, Hal Foster ou Arthur Danto, la citation et l'emprunt sont au centre des définitions du postmodernisme ${ }^{30}$. «L'art contemporain se définit entre autres choses par le fait que le passé est à disposition des artistes et qu'ils peuvent l'utiliser à leur guise ${ }^{31}$. » Il ne s'agit pas là d'un postmodernisme aveugle, pour pasticher Scarpetta: Godbold ne met pas toute représentation sur un plan d'égalité, il n'aplatit pas la différence entre le référentiel et le connotatif. En choisissant un dispositif de superposition, il met en tension deux types de représentation et en bouleverse les propriétés. Ainsi toute idée de valeur esthétique ne disparaît pas. Quatrièmement, le renoncement à la pureté moderne implique un renoncement à la logique de l'œuvre, à sa consistance. L'ordre symbolique, où les différences sont effacées et condensées en un signe, est remplacé par l'ordre allégorique qui est fondé sur l'hybridité, la superposition d'éléments textuels divers ${ }^{32}$. À l'hybridité de la forme correspond l'instabilité du sens et l'équivocité du langage. Dans The ONLY reality, un dessin à l'encre brune sur papier-calque représente un homme en bure agenouillé. À travers cette feuille, on voit une carte portant des marques attestant normalement son authenticité : le numéro 826 indique que le document fait partie d'un archivage ordonné, l'écriture à la plume semble ancienne, le texte est purement descriptif. Sous l'image, David Godbold a dactylographié le texte suivant: «At this very moment these words are the only reality that binds us together. That's the way with words, they conspire to form sentences and get out of control». Godbold souligne ici le manque de fiabilité du langage, la manipulation et l'illusion qu'il engendre.

De même que les travestissements burlesques en littérature insinuaient une critique de la fiction, les distorsions visuelles de Godbold initient une critique de la représentation artistique. Cette auto-critique est caractéristique du burlesque, de la parodie mais aussi $\mathrm{du}$ postmodernisme. Dominique Bertrand souligne que l'esthétique burlesque s'accommode souvent d'un méta-discours : « le burlesque mêle l'exaltation de l'art et de l'artiste à sa perpétuelle auto-dérision ${ }^{33}$. » Ainsi, l'auteur burlesque tourne en dérision la sacralisation excessive de la poésie. « Le burlesque remet en cause plus profondément une esthétique de la mimesis: l'exagération comique affranchit la représentation des contraintes usuelles du référent et du sens, induisant un primat de la fantaisie du créateur $^{34}$. " La déconstruction de l'image dans le travail de Godbold l'affranchit de la mimesis et induit une réflexion sur les dispositifs sémiotiques et institutionnels qui construisent l'œuvre d'art. La composition est donc une méta-icône.

17 Margaret Rose, dans Parody/Meta-fiction identifie la parodie à une forme de métafiction et lui attribue une relation particulière à la réflexivité35. Dans Parody : Ancient, Modern, and Postmodern ${ }^{36}$, Rose avance que la parodie postmoderne en littérature renoue avec le comique et la métafiction qui était à l'origine du genre. Elle emploie le terme de 
refunctioning qui vient du terme de Brecht, umfunktionerung, désignant le réemploi moderne de textes littéraires anciens. Ce terme pourrait de la sorte définir les réemplois modernes de thèmes iconographiques anciens par Godbold. Rose considère la parodie comme «une archéologie réflexive du texte, dans laquelle les conditions épistémologiques, historiques et sociales de la composition et de la réception des textes

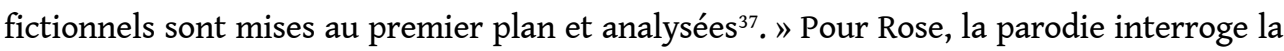
capacité de l'œuvre littéraire ou visuelle à représenter la réalité et à imiter, question posée par la modernité. Ces propos ne peuvent qu'évoquer un dessin de Godbold intitulé The modernity Problem, dans lequel l'allégorie de la modernité est aveuglée et enchaînée. Avec auto-dérision, Godbold légende un autre dessin «I hope you don't believe in art too much, it's bad for your health and obscures our view from other possibilities». La présence de la scène religieuse incite à interpréter la légende comme une critique de la propagande visuelle de la Contre-réforme. Godbold interroge également la référentialité du langage et les codes de composition. Dans une œuvre intitulée Institutional Means of Representation, le texte d'arrière-plan nous donne à lire : «classical narrative realistic text ». Un autre dessin est intitulé «the power of endo-diegetic focalisation ». La métaréférentialité est clairement affichée. Or Hal Foster avance que si le postmodernisme est référentiel, il ne renvoie qu'à la problématique de l'activité référentielle ${ }^{38}$. L'un des premiers théoriciens du postmodernisme, Charles Jencks, explique quant à lui que «le trope postmoderne est le commentaire ironique d'un commentaire qui met en exergue la distance ; un mythe nouveau par trois fois séparé de son rituel originel ${ }^{39}$. " L'œuvre de Godbold est un cas d'école.

18 À ce stade de notre réflexion, il semble nécessaire de revenir sur un élément matériel central au dispositif de Godbold : le calque. L'utilisation du calque est un procédé à la fois parodique et postmoderne qui joue sur la littéralité de la superposition. Linda Hutcheon, qui étudie la parodie dans l'art, note que « l'ironie et la parodie [...] opèrent toutes deux sur deux niveaux, un niveau de surface primaire en premier plan, un niveau secondaire et implicite en second plan. Ce dernier niveau, dans les deux cas, tire sa signification du contexte dans lequel il se trouve. La signification ultime du texte ironique ou parodique réside dans la superposition des deux niveaux, dans une sorte de double exposition (au sens photographique du terme) textuelle. $»^{40}$ La superposition évoquée ici prend un sens on ne peut plus littéral dans le travail de Godbold. Les différents plans de la parodie, l'hypo et l'hyper texte sont matérialisés grâce à l'emploi de calque et la stratégie parodique se trouve conséquemment exhibée. Qui plus est, si, comme l'affirme Emelina, "le comique, c'est l'envers du sérieux sous toutes ses formes " ${ }^{41}$, l'envers de tous les genres, alors le calque est l'envers, la norme retournée. Le calque matérialise la nécessaire dualité, voire duplicité, sur laquelle repose le comique. Enfin, à propos du burlesque, Dominique Bertrand parle d'un «principe de déconstruction généralisée, faisant éclater les genres traditionnels ou brouillant délibérément leurs frontières usuelles $^{42}$. $\gg$ Or, le calque réalise la déconstruction, il rend visible la déconstruction et devient méta-référentiel. Étant donné l'étymologie grecque du terme "parodie», $\pi \alpha \rho \omega \delta i ́ \alpha$, le dispositif choisi par Godbold, plus encore que l'hétérogénéité des composants, des registres et des genres, semble pertinent. 


\section{Irrespect esthétique ou dénonciation satirique?}

19 À propos de The Unreliable Narrator, le philosophe David Carrier a écrit: "Comme Baudelaire, dont le satanisme le captive, [Godbold] est fasciné par le catholicisme tout en faisant preuve envers lui de beaucoup d'irrespect. "La religion est un grand (mais explosif) théâtre de comédie » confie l'artiste. La religion est sans doute le grand sujet dans son œuvre mais il répond au catholicisme en termes purement esthétiques ${ }^{43}$. " Cette lecture de l'œuvre de Godbold mérite d'être réexaminée. La parodie chez Godbold est-elle exempte de toute tonalité satirique? Rappelons que si la parodie implique un regard critique sur la littérature, ou l'art, la satire quant à elle est un discours critique sur la société. Elle s'attaque aux défauts moraux, politiques d'une époque.

Dans plusieurs compositions, Godbold évoque de manière satirique le pathos mis à l'honneur dans rhétorique visuelle religieuse en insinuant dans l'image des références à la violence. La Contre-Réforme visait à rendre le divin tangible en faisant appel aux émotions et aux sens de la population. L'œuvre d'art était alors un instrument de propagande, le support d'une idéologie qui se cachait derrière un sensualisme nouveau. Au bas d'un dessin représentant la Trinité, Godbold a inscrit : «A sugar-coated message, stuffed into an allegorical drama». Certains dessins évoquent le poids de l'éducation religieuse et la violence des guerres saintes. Dans I'm Doing Much Better Now than I Have Ever Done Before, le pape tient un christ en croix d'une main et écrase la tête d'un jeune homme du pied droit. Un autre dessin, inspiré du massacre des Innocents, est légendé « increase the peace ». Dans l'œuvre de Godbold, se croisent un héritage dadaïste mâtiné de burlesque et une préoccupation tout irlandaise pour la religion. Son irrévérence n'est pas sans rappeler celle dont font preuve J.M. Synge, dans The Well of the Saints, ou bien Stewart Parker dans Pentecost. Des saints en extase ou ivres, des saintes larmoyantes, des martyrs ou des tortionnaires peuplent les œuvres hérétiques de Godbold et semblent céder à leur libido. Dans plusieurs planches, Godbold attaque l'Eglise en usant d'ironie. Dans I Only Know What I Believe, la légende de l'image est une distorsion de I only believe what I see; à une représentation tronquée de la Cène est apposée la légende "contains some moderate violence and occasional scary moments "; dans But Sadder, les mots «yoga is back» sont imprimés sous le christ aux stigmates. Parfois, Godbold élargit sa critique du discours au domaine politique. Increase your Emotional Range juxtapose un dessin à l'encre bleue à la manière des maîtres anciens et une page de livre contemporain portant la tête de chapitre suivante : Television : instrument of the state ? Godbold établit un parallèle entre le discours religieux, la rhétorique baroque et l'influence des grands médias.

21 La spécificité du dispositif visuel créé par Godbold permet la mise en tension des éléments divers qui constituent la parodie burlesque. Si, d'ordinaire, l'hypotexte de la parodie est présent en filigrane même s'il est masqué en partie par l'hypertexte, dans les compositions de Godbold, il est aisément perceptible. Equivalent visuel du zeugme, dont l'effet comique est avéré, le calque explicite la démarche parodique et la posture postmoderne. La nature ludique des parodies appuie le discours critique. L'œuvre de Godbold tend en effet à s'inscrire dans un postmodernisme lucide qui ne s'affranchit pas aisément de son rôle au sein de la société. Il semble en outre que le rire, procédé rhétorique servant aussi à convaincre, soit le moyen par lequel l'artiste et le spectateur revisitent à la fois la modernité du ready-made subversif et le pastiche postmoderne. 


\section{NOTES}

1. Marielle Macé, Le Genre Littéraire, Paris, GF Flammarion, 2004, p. 121.

2. Parody and Decadence, cité par Daniel Sangsue, La relation parodique, Paris, José Corti, coll. « Les Essais », 2007, p. 89.

3. Jean Emelina, Le Comique, essai d’interprétation générale, Sedes, Paris, Présences Critiques, 1991, p. 30.

4. Emelina, Ibid., p. 39.

5. Mikhaill Bakhtine, Esthétique et théorie du roman, Paris, Gallimard, trad. D. Olivier, 1978, p. 130.

6. Daniel Sangsue, op. cit., p. 74.

7. Ronald Schmitt, « Deconstructive comics ", Journal of Popular Culture, n² 25, 1992, p. 157-58.

8. Roland Barthes, S/Z, Paris, Points Seuil, 1970, p. 7.

9. Mikhail Bakhtine, op. cit., p. 122.

10. Ibid., p. 176-178.

11. Michele Hannoosh, Parody and Decadence, Laforgue's Moralités Légendaires, Colombus, the Ohio State University Press, 1989, cité dans Daniel Sangsue, op. cit., p. 87.

12. "Le mot 'burlesque' dans les dictionnaires de langue contemporains", dans Poétiques du burlesque, Actes du colloque international du CRLMC, Université Blaise Pascal, Paris, Honoré Champion, 1998, p. 33.

13. David Carrier, « David Godbold's Recent Drawings », dans Godbold, David, Once it was a lie, now it's the truth..., Dublin, Kerlin Gallery, 2005, p. 6.

14. Daniel Sangsue, op. cit., p. 77.

15. Henri Benac, Dictionnaire des Idées Littéraires, Paris, Hachette, 1998, p. 368.

16. Jean-Marc Defays, «Le burlesque et la question des genres comiques", dans Poétiques du burlesque, op. cit., p. 42.

17. Dominique Bertrand, « Poétiques du burlesque », dans Poétiques du burlesque, op. cit., p. 12.

18. Guy Scarpetta, L'Artifice, Paris, Grasset, coll. Figures, 1988, p. 131.

19. Dominique Bertrand, op. cit., p. 9-24.

20. Dominique Bertrand, op. cit., p. 21.

21. Jean Emelina, «Comment définir le burlesque? », dans Poétiques du burlesque, op. cit.,p. 58.

22. Ibid., p. 64.

23. Ibid., p. 61.

24. Ibid.,p. 16.

25. Jean-Marc Defays, op. cit., p. 43.

26. Ibid., p. 45.

27. Rosalind Krauss, «Expanded Field», October, n 8, printemps 1979, p. 42. Traduction de l'auteur.

28. Roland Barthes, Le Degré Zéro de l'Écriture, Paris, Seuil, 1953.

29. Ce fascicule fut reproduit dans Circa, $\mathrm{n}^{\circ}$ 84, été 1998.

30. Hal Foster, « Re-post ", dans Brian Wallis, Art After Modernism, Rethinking Representation, New York, Museum of Contemporary Art et David R. Godine, 1984, p. 189-202.

31. Arthur Danto, L'Art contemporain et la clôture de l'histoire, Paris, Seuil, 2000, p. 30.

32. "The Allegorical Impulse: Towards a Theory of Post-modernism ", Art After Modernism, op.cit., p. 235.

33. Ibid., p. 24.

34. Ibid., p. 24. 
35. Margaret Rose, Parody/Meta-fiction, Londres, Croom Helm, 1979, cité par Daniel Sangsue, op. cit., p. 76.

36. Cambridge University Press, 1993.

37. Margaret Rose, op. cit., p. 13.

38. Hal Foster, op.cit., p. 196.

39. Charles Jencks, What is Postmodernism?, Londres, Academy Éditions, 1996, p. 41.

40. Linda Hutcheon, «Ironie et parodie : stratégie et structure ", trad. P. Hamon, Poétique 36, 1978, p. 472- 73.

41. Jean Emelina, op. cit., p. 170.

42. Dominique Bertrand, op. cit., p. 18.

43. David Carrier, The Aesthetics of Comics, University Park, Pennsylvania University Press, 2000, p. 69. Traduction de l'auteur.

INDEX

Mots-clés : Godbold (David), comique, burlesque, beaux-arts, parodie

\section{AUTEUR}

\section{VALÉRIE MORISSON}

Université de Dijon, Centre Interlangues TIL, EA 4182. 